FTUAM-93/14

May, 1993

\title{
STRING VARIATIONS ON KALUZA-KLEIN COSMOLOGY
}

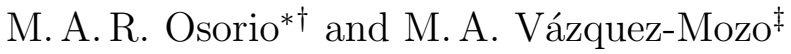 \\ Departamento de Física Teórica C-XI \\ Universidad Autónoma de Madrid \\ 28049 Madrid, Spain
}

\begin{abstract}
We study the cosmological solutions of the two-dimensional Brans-Dicke equations considering a gas of $c=1$ strings in $S^{1} \times \mathbf{R}$ as the source of the gravitational field. We also study the implications of the $R$-duality invariance on the solutions. To this purpose we conjecture that, as it happens for massless fields in finite boxes, the free energy of a gas of massless string excitations is not given by the corresponding toroidal compactification.
\end{abstract}

\footnotetext{
${ }^{*}$ E-mail addresses: OSORIO@VM1.SDI.UAM.ES and OSORIO@MADRIZ1.FT.UAM.ES.

${ }^{\dagger}$ Address after June 1st. 1993, Departamento de Física, Facultad de Ciencias, Universidad de Oviedo, Avda. Calvo Sotelo s/n, E-33007 Oviedo, Spain.

$\ddagger$ E-mail addresses: MAVAZ@VM1.SDI. UAM.ES and VAZQUEZ@MADRIZ1.FT.UAM.ES.
} 


\section{Introduction}

In a previous paper [1] we have studied the implications of the presence of compactified spatial dimensions (or equivalently, the non-trivial topology of the space-time) on the cosmological solutions of a two-dimensional toy model coupled to Brans-Dicke gravity. There we found that the only observable effect of the closed dimension appears in the thermal history. The universe with compactified dimensions gets cold more slowly than in the open space case. The dynamical evolution, on the contrary, remains exactly the same. The reason for this is that the introduction of non-trivial topology in the space does not modify the equation of state associated with the (field-theoretical) matter filling the universe.

Now we would like to study the cosmological solutions to the Brans-Dicke equations when the source of the gravitational field is a gas of strings living in a two-dimensional target space. From the very beginning we face a problem which is that of determining the correct expression of the free energy. If we take as true the conjecture of [2] in which the free energy is naively identified with the toroidal compactification in $S^{1} \times S^{1}$, the only remnant question to clarify is that of identifying the time coordinate. If we restrict our interest up to one loop in the worldsheet the question is irrelevant since the space dimension is compact and $R^{(2)}=0$, then this is only a problem at higher genus $(g \geq 2)$. If we took the Liouville field as the time coordinate its coupling to the world-sheet curvature would imply that only discrete values of the temperature are allowed [2]. On the contrary, based on aesthetical grounds, it looks more appealing to us to take the Liouville field as a spatial coordinate and then the length of the target coordinate of the $c=1$ string is $\beta$. This leads to a picture in which the string interaction produces a space whose length is quantized.

Following the conjecture of [2], an expression for the Helmholtz free energy is gotten which reproduces the partition function of the $c=1$ non-critical string on a two-dimensional target torus [7] (the concrete relation is that the sum over world-sheet surfaces equals $-\beta F(\beta)$ )

$$
F(\beta, L)=\frac{1}{\beta} \ln \left(\frac{L}{\sqrt{\alpha^{\prime}}}\right)+\frac{2}{\beta} \ln \left[\eta\left(i \frac{\beta L}{4 \pi^{2} \alpha^{\prime}}\right) \eta\left(i \frac{L}{\beta}\right)\right],
$$

where $L$ is the length of the spatial compactified dimension and $\eta(\tau)$ is the Dedekind $\eta$ function. The equation of state is

$$
\rho-p=\frac{1}{\beta L}-\frac{1}{12 \pi \alpha^{\prime}} E_{2}\left(i \frac{\beta L}{4 \pi^{2} \alpha^{\prime}}\right)
$$

with $E_{2}(\tau)$ the Eisenstein series. In this case we have some extra symmetries that are absent in the field-theoretical case, namely, the thermal partition function $Z(\beta, L)$ enjoys both $\beta$ and 
space-time duality

$$
Z(\beta, L)=Z\left(\frac{4 \pi^{2} \alpha^{\prime}}{\beta}, L\right)=Z\left(\beta, \frac{4 \pi^{2} \alpha^{\prime}}{L}\right)
$$

Since the Einstein-Hilbert-Brans-Dicke action

$$
S=\int d^{2} x \sqrt{-g}\left[\Phi(R-2 \Lambda)-\frac{\omega}{\Phi} \nabla_{\mu} \Phi \nabla^{\mu} \Phi\right]
$$

with the Friedmann-Robertson-Walker ansatz for the metric

$$
d s^{2}=-d t^{2}+L^{2}(t) d \xi^{2}
$$

and a space-independent Brans-Dicke field $\Phi(t)$ is invariant under the duality replacement

$$
L \rightarrow \frac{C}{L}, \quad \Phi \rightarrow \frac{L^{2}}{C} \Phi,
$$

with $C$ any constant, we see that the whole action (fields + matter) is invariant under the space-time duality transformation

$$
L \rightarrow \frac{4 \pi^{2} \alpha^{\prime}}{L}, \quad \Phi \rightarrow \frac{L^{2}}{4 \pi^{2} \alpha^{\prime}} \Phi .
$$

This is not true for $\beta$-duality. The reason is that only the matter action depends on the temperature and it transforms non-trivially under duality. Then the changes in the action of the matter induced by the duality transformation cannot be undone by the variation of the Einstein-Hilbert-Brans-Dicke action since this does not depend on $\beta$. The result is that the equations governing the dynamics of our universe are invariant under space-time duality but not under $\beta$-duality.

It seems then that $\beta$-duality does not survive the coupling to the Einstein-Hilbert-BransDicke action. Furthermore, what is really a problem is that (11) is positive definite for some region in the $\beta-L$ plane and actually it suffers from a fatal disease, the entropy is negative and divergent as $\beta \rightarrow \infty$. The only possible conclusion is that the partition function for the $c=1$ model on a torus, given by the integral on the fundamental region of the modular group of the solitonic contribution, does not coincide with minus the logarithm of the thermal partition function $Z(\beta, L)$. This is not a surprise because we have found the same situation for massless free fields in $S^{1} \times \mathbf{R}$ 固 and, after all, the $c=1$ model can be effectively described by a massless scalar field in two dimensions [3]. It is worth noticing that (11) can be written

$$
F(\beta, L)=\frac{1}{\beta} \ln \left(\frac{\beta}{\sqrt{\alpha^{\prime}}}\right)+F_{B}(\beta, L)+F_{B}\left(\beta, \frac{4 \pi^{2} \alpha^{\prime}}{L}\right)
$$

\footnotetext{
${ }^{1} L$ is the length of $S^{1}$
} 
where $F_{B}(\beta, L)=(2 / \beta) \ln \eta(i \beta / L)$ is the free energy of a massless boson in $S^{1} \times \mathbf{R}[1]$. Our proposal is that dropping the first term which depends only on $\beta$ we get the correct result for the one-loop free energy of the $c=1$ model in $S^{1} \times \mathbf{R}$. The price we have to pay is to renounce to $\beta$-duality. Nevertheless, we cure the maladie.

The plan of the paper is as follows. In section 2 we will study the main features of the thermodynamics of the critical two-dimensional string in $S^{1} \times \mathbf{R}$ and its relationship with a bosonic field living in the same space. In section 3 we will present the cosmological solutions to the Brans-Dicke equations. Finally in section 4 we will summarize the conclusions.

\section{The thermodynamics of the string in $S^{1} \times \mathbf{R}$}

The quantization of the bosonic string in arbitrary space-time dimension is a hard problem that still remains unsolved in general. The reason is that away from the critical dimension $(d=26)$ there is an anomaly associated with the conformal invariance of the string action [4. Then the conformal factor of the metric (the Liouville field) becomes a dynamical field that has also to be quantized. The quantization of the Liouville theory can be made in the particular case of the $c=1$ conformal field theory coupled to two-dimensional gravity [5]. In this case, after identifying the Liouville field as a new coordinate and with a suitable dilatonic background, the $c=1$ string can be reinterpreted as a critical two-dimensional string theory [6].

We are going to consider that the matter content of the universe is given by a gas of these two-dimensional critical strings. If we compactify the spatial coordinate in a circumference of radius $R=L / 2 \pi$, and take (1), i.e., the result of the toroidal compactification as computed in [7] (cf. [8, 2]), as the free energy we see that our thermodynamics enjoys both space-time and $\beta$-duality,

$$
F(\beta, L)=F\left(\beta, \frac{4 \pi^{2} \alpha^{\prime}}{L}\right)=\frac{4 \pi^{2} \alpha^{\prime}}{\beta^{2}} F\left(\frac{4 \pi^{2} \alpha^{\prime}}{\beta}, L\right) .
$$

In addition to this, $-\beta F(\beta, L)$ is invariant under the interchange $\beta \leftrightarrow L$, as it should be if the free energy is gotten from the toroidal compactification in $S^{1} \times S^{1}$.

Let us review the main thermodynamical properties that can be extracted from (11). The first important thing to mention is that $F(\beta, L)$ is not always negative. One can think that, after all, being the result of a regularization procedure some arbitrary term of the form constant/ $\beta$ can be added. Physically, adding this term is equivalent to tuning the value of the canonical entropy at zero temperature $(\beta=\infty)$. Then one can try to fix the constant to get zero entropy at zero temperature. From the very beginning this is not a legitimate manipulation because in quantum statistical mechanics the degeneration of the vacuum is what it is. But, we do not even need to think about this problem because fixing the entropy at zero temperature to 
zero by adding that term is impossible since the entropy behaves as minus $\ln \beta$ as $\beta \rightarrow \infty$ (see below).

A similar phenomenon is observed in critical strings at finite temperature [9] where the dual phase has bounded negative entropy. Here one is also tempted to add the adequate constant to set the entropy positive since at least it is only a finite constant. But one must be careful because standard texts in thermodynamics and statistical mechanics (see for example [10]) teach us that the entropy measures the number of states at a given energy that can be excited. But in String Theory it seems that at the self-dual point new degrees of freedom are excited [11] that kill the low energy field-theoretical ones. Let us concentrate for a moment in the particular case of the supersymmetric heterotic string at finite temperature [12, 13]. $\beta$-duality implies that at high temperature there are degrees of freedom which act still as fermions so as to recover a high temperature version of supersymmetry. In standard statistical mechanics it is precisely the equivalence between Bose, Fermi and Maxwell-Boltzmann statistics at high temperature and low density which fixes the additive constant in the entropy. Our conclusion is that all these things, namely, the statistics of the string itself and the physical degrees of freedom that it represents, are involved in the resolution of this problem that is a mystery to us. In the problem we have now at hand, by inspecting the expressions for the energy and the pressure, we can see that high temperature and low density can correspond either to a stringy or a field-theoretical regime.

The canonical entropy obtained from (11) is

$$
\begin{aligned}
S(\beta, L) & =-\frac{\beta L}{12 \pi \alpha^{\prime}} E_{2}\left(i \frac{\beta L}{4 \pi^{2} \alpha^{\prime}}\right)+\frac{\pi L}{6 \beta} E_{2}\left(i \frac{L}{\beta}\right)-\beta F(\beta, L) \\
& =1-\ln \frac{\beta}{\sqrt{\alpha^{\prime}}}+S_{B}(\beta, L)+S_{B}\left(\beta, \frac{4 \pi^{2} \alpha^{\prime}}{L}\right),
\end{aligned}
$$

where

$$
S_{B}(\beta, L)=-2 \ln \eta\left(i \frac{\beta}{L}\right)-\frac{\pi \beta}{6 L} E_{2}\left(i \frac{\beta}{L}\right)
$$

is the entropy for a massless bosonic field. From this expression we see that when $\beta$ goes to infinity the entropy diverges as minus $\ln \beta$ since $S_{B}$ is zero in that limit. The problem is not that the entropy is negative, but the fact that it is unbounded. $\beta$-duality leads also to an intriguing relationship between the Helmholtz free energy and the average of the entropy, namely,

$$
-\beta F(\beta, L)=\frac{1}{2}\left[S(\beta, L)+S\left(\frac{4 \pi^{2} \alpha^{\prime}}{\beta}, L\right)\right] .
$$

Another consequence of $\beta$-duality is that the energy density $\rho(\beta, L)$ vanishes at the self-dual temperature $\beta=2 \pi \sqrt{\alpha^{\prime}}$ for every value of the volume. Similarly, $L$-duality implies the vanishing 
of the pressure for $L=2 \pi \sqrt{\alpha^{\prime}}$ and any value of $\beta$. This means that at the self-dual temperature and the self-dual size we have $\rho=p=0$. We will see in section 3 that this particular situation would define a static euclidean universe.

The main question is that of the existence of any connection between $F(\beta)$ given by (1) and any quantum field theory. As mentioned in [1], (1) does not coincide with the free energy of a massless scalar boson. Finding a relationship with fields is equivalent to distinguishing a stringy regime. The presence of $\alpha^{\prime}$ induces us to identify the field-theoretical regime as that arising in the limit $\beta L \gg 4 \pi^{2} \alpha^{\prime}$, to get

$$
F(\beta, L) \rightarrow-\frac{L}{24 \pi \alpha^{\prime}}+\frac{1}{\beta} \ln \left(\frac{\beta}{\sqrt{\alpha^{\prime}}}\right)+\frac{2}{\beta} \ln \eta\left(i \frac{\beta}{L}\right),
$$

since in this approximation we have that

$$
-\frac{1}{\beta}\left[\frac{\beta L}{24 \pi \alpha^{\prime}}+\ln \left(\frac{\beta}{\sqrt{\alpha^{\prime}}}\right)\right] \sim-\frac{L}{24 \pi \alpha^{\prime}},
$$

we finally obtain the free energy for a massless boson in $S^{1} \times \mathbf{R}$ with an additional vacuum energy given by $-L /\left(24 \pi \alpha^{\prime}\right)$. The corresponding equation of state is $p=\rho+L /\left(12 \pi \alpha^{\prime}\right)$.

On the other hand we can also identify an ultrastringy regime in which $\beta L \ll 4 \pi^{2} \alpha^{\prime}$. In this limit the Helmholtz free energy takes the form

$$
F(\beta, L) \rightarrow-\frac{2 \pi^{3} \alpha^{\prime}}{3 \beta^{2} L}+\frac{2}{\beta} \ln \eta\left(i \frac{L}{\beta}\right)
$$

which gives rise to the equation of state $p=\rho-4 \pi^{3} \alpha^{\prime} /\left(3 \beta^{2} L^{2}\right)$. So, in this regime, the string cannot be described in terms of a quantum field theory, as it must be if string theory is something more than ordinary quantum field theory.

We have seen that the free energy (11) suffers from a severe problem, namely, the canonical entropy diverges when $\beta$ goes to infinity. This is a consequence of the presence of the term

$$
\frac{1}{\beta} \ln \left(\frac{\beta}{\sqrt{\alpha^{\prime}}}\right)
$$

in the free energy which ensures $\beta$-duality and the invariance of $\beta F(\beta, L)$ under the replacement $\beta \leftrightarrow L$. In order to solve the problems with (11) we propose that the correct result for the free energy of the $c=1$ string at finite temperature is obtained by dropping the term containing $\ln \left(\beta / \sqrt{\alpha^{\prime}}\right)$ in $($ 回 $)$, namely

$$
\hat{F}(\beta, L)=F_{B}(\beta, L)+F_{B}\left(\beta, \frac{4 \pi^{2} \alpha^{\prime}}{L}\right)=\frac{2}{\beta} \ln \eta\left(i \frac{\beta}{L}\right)+\frac{2}{\beta} \ln \eta\left(i \frac{\beta L}{4 \pi^{2} \alpha^{\prime}}\right) .
$$


The new equation of state is

$$
\rho-p=-\frac{1}{12 \pi \alpha^{\prime}} E_{2}\left(i \frac{\beta L}{4 \pi^{2} \alpha^{\prime}}\right) .
$$

That $\hat{F}$ gives a positive definite canonical entropy is a trivial exercise since it is the sum of the entropies for two bosonic fields. What is more important is to check that using (17) we can recover the field-theoretical regime when $\beta L \gg 4 \pi^{2} \alpha^{\prime}$. Using the expression of the Dedekind $\eta$-function we get

$$
\hat{F}(\beta, L) \rightarrow-\frac{L}{24 \pi \alpha^{\prime}}+\frac{2}{\beta} \ln \eta\left(i \frac{\beta}{L}\right),
$$

i.e., we get the expression for a massless field with a vacuum energy, in accordance with the result (14) obtained from (1). In the ultrastringy regime $\left(\beta L \ll 4 \pi \alpha^{\prime}\right)$ the limit we get has the same form than the one obtained from (1),

$$
\hat{F}(\beta, L) \rightarrow-\frac{2 \pi^{3} \alpha^{\prime}}{3 \beta^{2} L}+\frac{2}{\beta} \ln \eta\left(i \frac{\beta}{L}\right) .
$$

Nevertheless, we have to use an aproximation similar to that in (14).

As we have pointed out, $\beta \hat{F}(\beta, L)$ does not present either $\beta$-duality or invariance under the replacement $\beta \leftrightarrow L$. Nevertheless, as mentioned in sec. $1, \beta$-duality does not survive the coupling to Brans-Dicke gravity so it seems that this is not a fundamental symmetry to preserve. With respect to the breaking of the invariance under the interchange of $\beta$ and $L$ this is a consequence of the fact that the proposed free energy is not obtained from the toroidal compactification in $S^{1} \times S^{1}$. We have shown [1] that the same breakdown of the equivalence between the Helmholtz free energy and the toroidal compactification happens when studying massless fields living in compact spaces, in particular in $S^{1} \times \mathbf{R}$. What we claim is that this is what happens when considering a two-dimensional string in $S^{1} \times \mathbf{R}$ : the Helmholtz free energy is not given by the toroidal compactification. We state that the free energy of the twodimensional string can be interpreted as the one corresponding to two massless fields in $S^{1} \times \mathbf{R}$, one living in a circumference of length $L$ and the other one in a circumference of length $4 \pi^{2} \alpha^{\prime} / L$ (cf. [8]). Notice that (17) enjoys invariance under the duality transformation $L \rightarrow 4 \pi^{2} \alpha^{\prime} / L$. Since this is a symmetry of the Brans-Dicke action (with the ansatz (5) for the metric) this seems to be the preserved fundamental symmetry.

In the next section we will study the solutions to the Einstein-Hilbert-Brans-Dicke equations using either (1) or (17) for the Helmholtz free energy. 


\section{Cosmological solutions}

We are interested in the cosmological solutions of our stringy universe. Then we are going to consider our string propagating in the presence of a background metric $g_{\mu \nu}$ and a background dilaton field $\Phi$. The condition for this background to define a CFT (to lowest order in $\alpha^{\prime}$ ) is given by the vanishing of the $\beta$-functions associated with the background fields. The equations so obtained for $g_{\mu \nu}$ and $\Phi$ can also result from using the action principle with the following action functional [2]

$$
S_{e f f}=\int d^{2} \xi \sqrt{-g}\left[\Phi\left(R+\frac{16}{\alpha^{\prime}}\right)+\frac{1}{\Phi} \nabla_{\mu} \Phi \nabla^{\mu} \Phi\right]
$$

which correspond to the Brans-Dicke action (困) with $\omega=-1$ and $\Lambda=-8 / \alpha^{\prime}$. The coupling to the matter can be made via the perfect-fluid energy-momentum tensor

$$
T_{\mu \nu}=(p+\rho) u_{\mu} u_{\nu}-p g_{\mu \nu}
$$

The equations for the background fields are then

$$
\begin{aligned}
-\frac{8}{\alpha^{\prime}} g_{\mu \nu} & =\frac{8 \pi}{\Phi} T_{\mu \nu}-\frac{1}{\Phi^{2}}\left(\nabla_{\mu} \Phi \nabla_{\nu} \Phi-\frac{1}{2} g_{\mu \nu} \nabla_{\sigma} \Phi \nabla^{\sigma} \Phi\right) \\
& +\frac{1}{\Phi}\left(\nabla_{\mu} \nabla_{\nu} \Phi-g_{\mu \nu} \square \Phi\right), \\
R+\frac{16}{\alpha^{\prime}} & =-\frac{1}{\Phi^{2}} g^{\mu \nu} \nabla_{\mu} \Phi \nabla_{\nu} \Phi+\frac{2}{\Phi} \square \Phi,
\end{aligned}
$$

together with the integrability condition imposed by the local conservation of the energymomentum tensor $\nabla_{\mu} T^{\mu \nu}=0$.

For the background metric we are going to use the Friedmann-Roberston-Walker ansatz (5) and a space-independent dilaton field $\Phi(t)$. In this case eqs. (23) and (24) can be rewritten as

$$
\begin{aligned}
2 \Phi^{2} \frac{\ddot{L}}{L}+\frac{16}{\alpha^{\prime}} \Phi^{2} & =\dot{\Phi}^{2}-2 \Phi \ddot{\Phi}-2 \Phi \dot{\Phi} \frac{\dot{L}}{L}, \\
\dot{\Phi}^{2}+2 \Phi \dot{\Phi} \frac{\dot{L}}{L} & =16 \pi \Phi \rho(\beta, L)-\frac{16}{\alpha^{\prime}} \Phi^{2}, \\
\Phi \ddot{\Phi}-\frac{1}{2} \dot{\Phi}^{2} & =-8 \pi \Phi p(\beta, L)-\frac{8}{\alpha^{\prime}} \Phi^{2},
\end{aligned}
$$

and the energy-momentum conservation

$$
\dot{\rho}(\beta, L)+\frac{\dot{L}}{L}[\rho(\beta, L)+p(\beta, L)]=0 .
$$

It is easy to check that eq. (25) is equivalent to the conservation of $T_{\mu \nu}$ so we can drop it and be left with eqs. (26), (27) and (28) that together with the equation of state determine our system in terms of $\Phi(t), L(t)$ and $\beta(t)$. 
Let us now make some comments about $L$-duality. We have seen that $\hat{F}(\beta, L)$ (and $F(\beta, L))$ are invariant under the replacement $L \rightarrow 4 \pi^{2} \alpha^{\prime} / L$. This implies that the energy density and the pressure transform according to

$$
\rho(\beta, L)=\frac{4 \pi^{2} \alpha^{\prime}}{L^{2}} \rho\left(\beta, \frac{4 \pi^{2} \alpha^{\prime}}{L}\right), \quad p(\beta, L)=-\frac{4 \pi^{2} \alpha^{\prime}}{L^{2}} p\left(\beta, \frac{4 \pi^{2} \alpha^{\prime}}{L}\right)
$$

It can be easily checked that these transformations for the energy density and the pressure, together with the transformation of the dilaton field $\Phi(t)$ make the system of differential equations (26), (27) and (28) invariant under $L$-duality (in fact, the equations combine among themselves). A pending problem is that of clarifying what is the dynamical meaning of this symmetry. It has been argued in [14, 2] that the duality property would imply an effective minimum length $L_{\text {min }}=2 \pi \sqrt{\alpha^{\prime}}$ for the universe. Nevertheless, in [14, the arguments leading to this conclusion lie upon the condition that one has to substitute the definition of the localized states in terms of the Fourier transform of the momentum modes when $L>L_{\min }$ (which are light in this regime), by the definition in terms of the Fourier transform of the winding modes when $L$ decreases below the self-dual length (which are the light states in this case). Nevertheless, the only conclusion one can extract from $L$-duality using equations (26)-(28) is that, for any solution $L(t), \Phi(t)$ and $\beta(t)$, the new set of functions

$$
L^{\prime}(t)=\frac{4 \pi^{2} \alpha^{\prime}}{L(t)}, \quad \Phi^{\prime}(t)=\frac{L^{2}(t)}{4 \pi^{2} \alpha^{\prime}} \Phi(t), \quad \beta^{\prime}(t)=\beta(t),
$$

is also a solution to (26)-(28). In fact, as we will see in a moment, there are solutions for which $L(t)$ goes through the self-dual length and ends at $L=0$. This kind of solutions exist when we take $F(\beta, L)$ as the free energy as well as when $\hat{F}(\beta, L)$ is chosen.

With respect to $\beta$-duality, the situation is somewhat different. The transformation property of $F(\beta, L)$ with respect to the duality replacement $\beta \rightarrow 4 \pi^{2} \alpha^{\prime} / \beta$ implies the following transformation rules for the energy density and the pressure

$$
\rho(\beta, L)=-\frac{4 \pi^{2} \alpha^{\prime}}{\beta^{2}} \rho\left(\frac{4 \pi^{2} \alpha^{\prime}}{\beta}, L\right), \quad p(\beta, L)=\frac{4 \pi^{2} \alpha^{\prime}}{\beta^{2}} p\left(\frac{4 \pi^{2} \alpha^{\prime}}{\beta}, L\right) .
$$

It is now clear from eqs. (26)-(28) that $\beta$-duality is broken by the coupling the two-dimensional dilatonic gravity. This is because, unless the case of $L$-duality, the geometric part of the action, i.e., $L(t)$ and $\Phi(t)$, do not transform under $\beta$-duality, so one cannot reabsorb the change induced in the energy density and the pressure by a change in the dynamical variables $L$ and $\Phi$.

When solving the classical equations of motion for the fields, there are a set of variables in terms of which the expressions simplify notably and which have a clear physical meaning. Let 
us define two new functions $u(t), v(t)$ in terms of $\beta(t)$ and $L(t)$

$$
u(t)=\frac{L(t)}{\beta(t)}, \quad v(t)=\frac{\beta(t) L(t)}{4 \pi^{2} \alpha^{\prime}} .
$$

Here $v$ is a kind of stringiness parameter; when $v \gg 1$ we are in what we called before the field-theoretical regime. In that case, since the string Helmholtz free energy (either $F$ or $\hat{F}$ ) reduces to the free energy for a massless boson in $S^{1} \times \mathbf{R}$ plus a vacuum energy, we expect the universe to behave as in the cases studied in [1] with the appropriate values for $\omega$ and $\Lambda$. On the other hand, the limit $v \ll 1$ corresponds to a ultrastringy regime in which the matter content of the universe is governed by the equation of state deduced in sec. 2 .

When studying the numerical solutions to (26)-(28) using the matter described by (17) one can find three different kinds of solutions depending on the initial conditions $u_{0}, v_{0}, \Phi_{0}$ and $\dot{\Phi}_{0}$. The first one corresponds to field-theoretical-like universes in which $v(t) \gg 1$ for every value of $t$. In fig. 1 we have plotted $L(t)$ for this class of solutions. The universe contracts from an infinite size and reaches a minimum length that, however, is much larger than the self-dual size. The dilaton field (fig. 2) grows and, after reaching a maximum, begins to decrease. In fig. 3 we have plotted the time evolution of the temperature for this universe. We see that, as it corresponds to a field-theoretical universe, the temperature drops to zero when the size of the universe goes to infinity (cf. the results gotten in ref. [1]).

A second kind of solutions arise when we consider less restrictive initial conditions. In this case we get a universe whose scale factor $L(t)$ comes from infinity and after reaching the self-dual length keeps on decreasing until it gets $L=0$ (fig. 田). The dilaton, on the other hand, grows monotonously as it is shown in fig. 5. The temperature, nevertheless, has a quite stringy behavior (fig. 6). For small $t$, when the universe is big, the temperature is low, which is consistent with a field-theoretical description. However, when the size of the universe gets below the self-dual size, the temperature, instead of continuing raising (as one expects from field theory) begins to decrease and it is zero when the universe reaches a null size. This behavior is a consequence of $L$-duality, because the entropy satisfies $S(\beta, L)=S\left(\beta, 4 \pi^{2} \alpha^{\prime} / L\right)$. Since the integrability condition $\nabla_{\mu} T^{\mu \nu}=0$ implies that the entropy is conserved, we have

$$
\frac{d}{d t} S(\beta, L)=0=\frac{\partial S}{\partial \beta} \dot{\beta}+\frac{\partial S}{\partial L} \dot{L}
$$

Being the scale factor $L(t)$ a monotonously decreasing function, we can parametrize the evolution of $\beta$ by $L$. Then, from the last relation we have

$$
\frac{d \beta}{d L}=\frac{\dot{\beta}}{\dot{L}}=-\frac{\partial S / \partial L}{\partial S / \partial \beta}[\beta(L), L]
$$


Now, implementing duality it is easy to check that

$$
\frac{d \beta}{d L}[\beta(L), L] \frac{d \beta}{d L}\left[\beta\left(\frac{4 \pi^{2} \alpha^{\prime}}{L}\right), \frac{4 \pi^{2} \alpha^{\prime}}{L}\right]<0,
$$

so this implies that $d \beta / d L=0$ if $L=2 \pi \sqrt{\alpha^{\prime}}$.

We have said above that the meaning of $L$-duality is that the solutions can be paired according to (30). When we apply the duality transformations to the second kind of solutions describing a collapsing universe, we get new solutions which closely resembles the time reversal of the original ones, i.e., we have a scale factor that starts at $L=0$ and grows monotonously, passing through the self-dual value, and reaching infinity in a finite time. Nevertheless, when we apply (30) to the first kind of solutions (the field-theoretical-like ones) we get a new class featuring a string that lives in the ultrastringy regime all the time. The scale factor $L(t)$ starts and ends at zero length and reaches a maximum value, much smaller than the self-dual one (fig. 7). With respect to the dilaton field we get a monotonously increasing $\Phi(t)$ very different from the field-theoretical type (fig. 8). The temperature, on the contrary, is the corresponding to the field-theoretical-like case (fig. 3).

One can ask oneself whether there is a solution describing a static universe. Imposing the condition $\dot{L}=\ddot{L}=0$ on eqs. (26)-(28) we find that there is one given by

$$
L(t)=2 \pi \sqrt{\alpha^{\prime}}, \quad \beta(t) \sim 2 \pi \sqrt{\alpha^{\prime}} \times 0.523522, \quad \Phi(t)=C \exp \left(i \frac{4 t}{\sqrt{\alpha^{\prime}}}\right),
$$

where the values of $L$ and $\beta$ are fixed by the requirement that the energy density and the pressure vanish. We dislike complex dilatonic fields since the $\sigma$-model dilaton is a real field. This can be fixed by performing a Wick rotation to imaginary time $t \rightarrow i t$. In that case we get a solution to Euclidean dilatonic gravity with constant scale factor. This solution can be interpreted as a kind of gravitational instanton interpolating between two universes, both at the self-dual size, one of which is in a strong coupling regime $(\Phi \ll 1)$ and the other one in a weak coupling one $(\Phi \gg 1)$ since we relate the string coupling constant with the vacuum expectation value of the dilaton field. Besides, as it is the case with most of the known gravitational instantons, this configuration of the fields renders the geometric part of the action zero. One can see that the static situation results from the action of the negative Casimir energy which compensates the positive contribution of the thermal motion to the internal energy.

Now we are going to study how all this structure of solutions changes when we substitute the Helmholtz free energy given by (17) by the one gotten from the toroidal compactification, i.e. (1). The first thing we can see is that, since in this case $F(\beta, L)$ recovers the field theory regime when $v \gg 1$, we are operatoralso going to have field-theoretical-like solutions of the type 
described above. On the other hand the ultrastringy solutions gotten from them by performing the duality transformation (30) are also to be present (let us recall that $F$ does enjoy $L$ duality as well as $\beta$-duality). An important point nevertheless is that now we have a kind of intermediate solutions of the type depicted in figs. 9 and 10. This intermediate (marginal) case sitting between the first (field-theoretical) and the second types can be gotten by making a fine-tuning of the initial conditions. The corresponding universe has a scale factor (see fig. 9) which comes from infinity and, after reaching the self-dual length, it stays at that value of $L$ for a long time and finally drops to $L=0$. Of course, by applying $L$-duality to (30) we get a similar exploding solution starting at $L=0$. The temperature now follows a curve similar to that of fig. 6, i.e., it is zero in both limits, but with a pronounced plateau (fig. 10). Besides, we also find those solutions describing a monotonously contracting (resp. expanding) universe ending (resp. beginning) at $L=0$ of the type described by figs. 땅.6. The instanton-like solution described above, is also present but now the existence of $\beta$-duality sets the temperature to the self-dual value.

In both cases (for $\hat{F}$ and $F$ ) the field equations are invariant under time reversal. This means that, together with the type of solutions described above we also have their time reversed ones which differ by the sign of $\dot{\Phi}_{0}$. All the plots have been made in units in which $\alpha^{\prime}=1$.

\section{Conclusions}

We have studied the cosmological solutions to dilatonic gravity using as the source of the gravitational field a gas of two-dimensional strings. The first thing we have found is that the expression of the Helmholtz free energy given by (11) and deduced in [7] presents an important problem; it gives a canonical entropy which is negative and diverges in the low temperature limit. As a solution we have proposed for the Helmholtz free energy another expression, (17), in which we drop the term which produces the problem. The principal feature of this new expression is that it breaks $\beta$-duality and, consequently, invariance under the interchange $\beta \leftrightarrow L$. The new expression can be interpreted as the free energy for two massless bosons one of which lives in a circumference of length $L$ and the other one in a circumference of length $4 \pi^{2} \alpha^{\prime} / L$ (cf. 8 ). This breaking of the equivalence between the toroidal compactification and the Helmholtz free energy can be traced back to the massless bosonic field in $S^{1} \times \mathbf{R}$ 迎. Since the only on-shell state of the $c=1$ string (despite of the topological discrete states, which do not contribute to the partition function [15]) is the center of mass which can be effectively described by a massless bosonic field [3], it seems to be natural to keep this equivalence at finite temperature. Indeed, the effective image of the $c=1$ string (or equivalently, the critical two-dimensional one) is that of its center of mass. If we look at it as a point on a cylinder, its hamiltonian would be given 
by $\hat{H}_{1}=\hat{m} / L$ where $\hat{m}$ is the operator whose eigenvalues are the momentum integer numbers of the string. On the other hand, since the string is an extended object it can wrap around the spatial compactified dimension and we have another contribution to its energy given by $\hat{H}_{2}=\hat{n} L /\left(4 \pi^{2} \alpha^{\prime}\right), \hat{n}$ being the winding number operator with integer eigenvalues $n$. Then, from a target-space point of view the dynamics of the string is governed by the hamiltonian

$$
\hat{H}=\frac{\hat{m}}{L}+\frac{\hat{n} L}{4 \pi^{2} \alpha^{\prime}}
$$

To compute the thermal partition function of the multi-string system we need to make a trace over the second quantized states $\left|m_{i}, n_{i}\right\rangle$ which are eigenvectors of the operators $\hat{m}_{i}$ and $\hat{n}_{i}$. Since the complete Hilbert space is the direct product of the winding and the momentum sectors the partition function factorizes and this is reflected in the fact that the Helmholtz free energy is the sum of two terms which correspond to two massless fields one in a space with length $L$ and the other one in the dual space. Now it is important to notice that the zero mode in both traces has to be suppressed. By the way, the partition function in the zero temperature limit computed in [3] can be interpreted as the partition function of two bosonic massless fields one at a temperature $T=1 / L$ and the second one at $T=L /\left(4 \pi^{2} \alpha^{\prime}\right)$

After studying the cosmological solutions, using both $F$ and $\hat{F}$ we arrive at several conclusions. First of all, we find that there is no dynamical rebound of the scale factor when reaching the self-dual size. Secondly we find that our string universe is not free from singularities. In fact, we have solutions that begin or end at $L=0$. At first sight this is quite surprising because they are absent in a field-theoretical universe (see [1]). But one of the claims in [14 is that windings prevent expansion and that is indeed what they do so as to get $L=0$. What we do not see is the suspected behaviour of the momentum states preventing the contraction. This effect would be the result of changing from the momentum modes when $L>2 \pi \sqrt{\alpha^{\prime}}$ to the winding modes when $L>2 \pi \sqrt{\alpha^{\prime}}$. In some sense we can say that the existence of this kind of singular solutions is implied by duality since the equivalence of $L=0$ and $L=\infty$ is a result of this symmetry.

It is really important to notice that when all the spatial dimensions are compact the thermal free energy is not given by the corresponding toroidal compactification if massless fields are involved. For example, this means that the free energy for the heterotic supersymmetric string with all the spatial dimensions compactified is not given by the toroidal compactification because the massless excitations of the string has to be treated with care. At least this is pretty clear from the analogue model in which we compute the free energy summing over the field content. Using this approach in a proper time representation of the free energy we know that the massless sector is not going to be correctly described. On the contrary, the modular invariant result for the associated toroidal compactification (see second reference in 
[11) does not present any divergence for any value of $\beta$ when $\operatorname{Im} \tau$ goes to infinity where $\tau$ is the standard one-loop modular parameter. What happens is that the connection between the modular invariant result and the proper time implementation of the analogue model is broken for any value of $\beta$. If we forget about this way of representing the analogue model we can try to implement it by directly writting the contribution of the massless sector as computed in [1] ] and then adding the contribution of the massive states in the proper time representation which is perfectly well defined. It seems that now the question about modular invariance is nonsense.

As a final comment, the qualitative effect of substituting $\hat{F}(\beta, L)$ by $F(\beta, L)$ is only to add the pseudo-statical solutions of fig. 9; we preserve the other three types of solutions discussed in sec. 3.

\section{Acknowledgements}

We thank J. L.F. Barbón for valuable discussions. We acknowledge finantial support by the CICyT Project No. AEN-90-0272. The work of M.A.V.-M. has also been partially supported by a Comunidad Autónoma de Madrid Predoctoral Fellowship.

\section{References}

[1] M. Osorio and M. Vázquez-Mozo, Variations on Kaluza-Klein Cosmology, FTUAM-93/13, 1993.

[2] A. Tseytlin and C. Vafa, Nucl. Phys. B372 (1992) 443.

[3] J. Polchinski, Nucl. Phys. B346 (1990) 253.

N. Sakai and Y. Tanii, Intern. J. Mod. Phys. A6 (1991) 2743.

M. Bershadsky and I. Klebanov, Phys. Rev. Lett. 65 (1990) 3088.

[4] A. Polyakov, Phys. Lett. 103B (1981) 207.

[5] F. David, Mod. Phys. Lett. A3 (1988) 1651.

J. Distler and H. Kawai, Nucl. Phys. B321 (1989) 509.

[6] P. Ginsparg and G. Moore, Lectures on 2D Gravity and 2D String Theory, YCTP-P23-92, LA-UR-92-3479, 1993 (hep-th/9304011).

[7] L. Dixon, V. Kaplunovsky and J. Louis, Nucl. Phys. B355 (1991) 649.

[8] H. Ooguri and C. Vafa, Nucl. Phys. B371 (1991) 469. 
[9] E. Alvarez and M. Osorio, Physica A 158 (1989) 449.

[10] R. Balescu, Equilibrium and non-equilibrium statistical mechanics, John Wiley and Sons, New York 1975.

[11] M. Osorio and M. Vázquez-Mozo, Phys. Lett. 280B (1992) 21.

M. Osorio and M. Vázquez-Mozo, Phys. Rev. D47 (1993) 3411.

[12] J. Atick and E. Witten, Nucl. Phys. B310 (1988) 291.

[13] E. Alvarez and M. Osorio, Nucl. Phys. B304 (1988) 327.

[14] R. Brandenberger and C. Vafa, Nucl. Phys. B316 (1988) 391.

[15] A. Polyakov, Mod. Phys. Lett. A6 (1991) 635.

I. Klebanov and A. Polyakov, Mod. Phys. Lett. A6 (1991) 3273. 


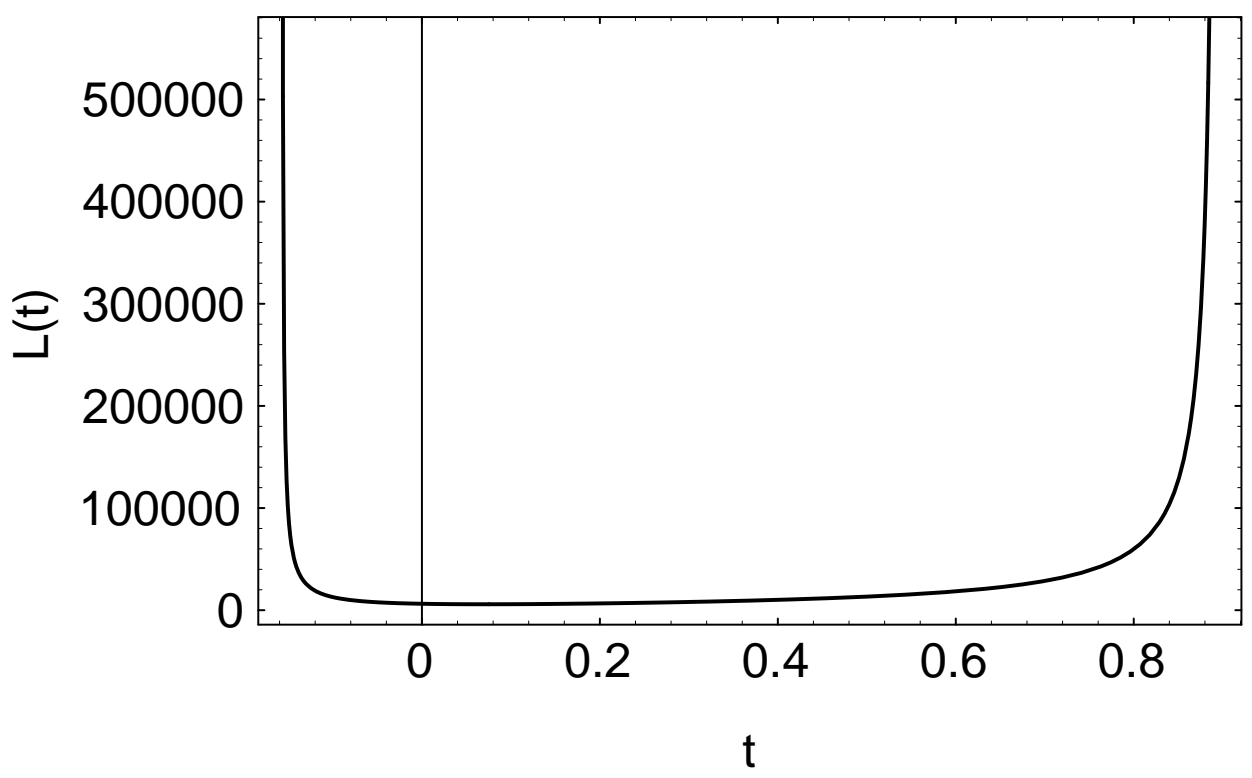

Figure 1: Scale factor $L$ vs. $t$ for $v \gg 1$. 


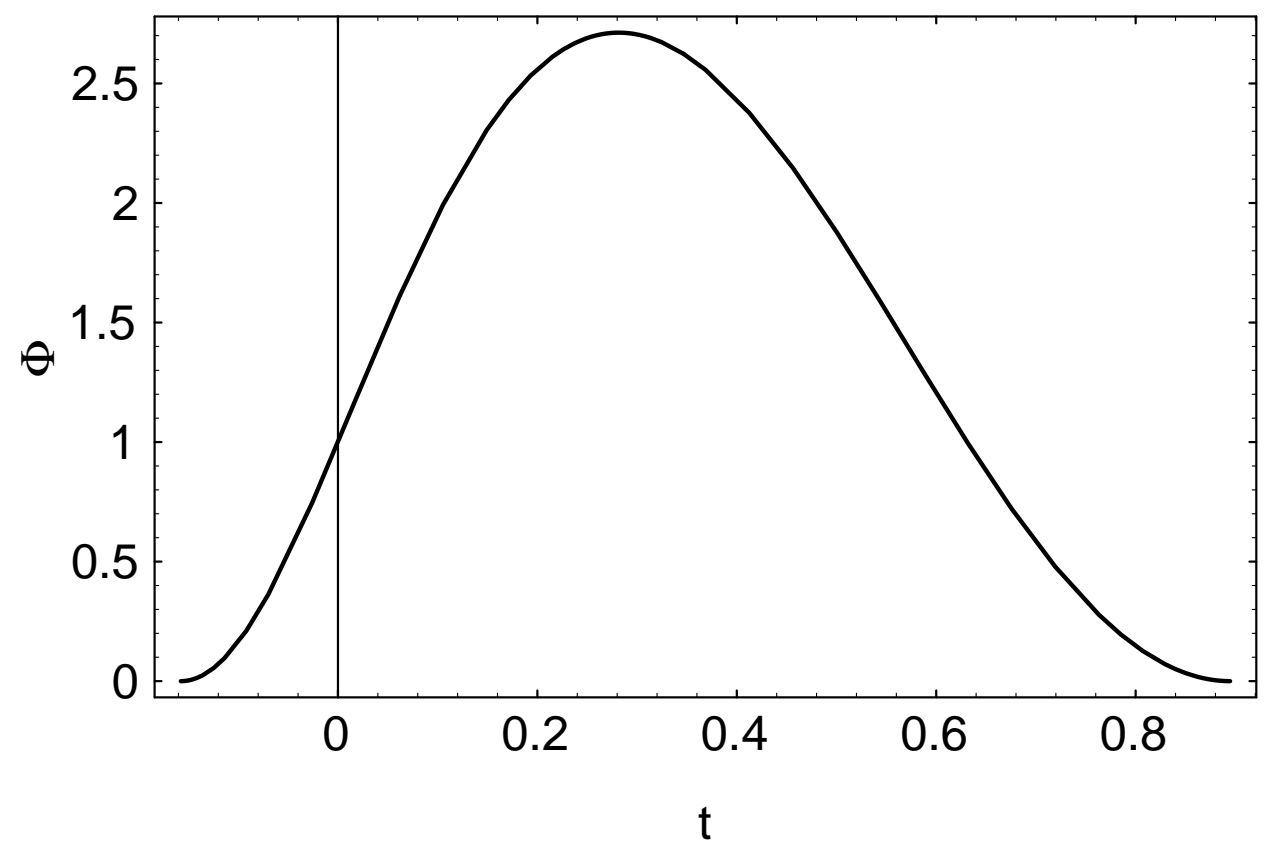

Figure 2: Dilaton vs. $t$ for $v \gg 1$.

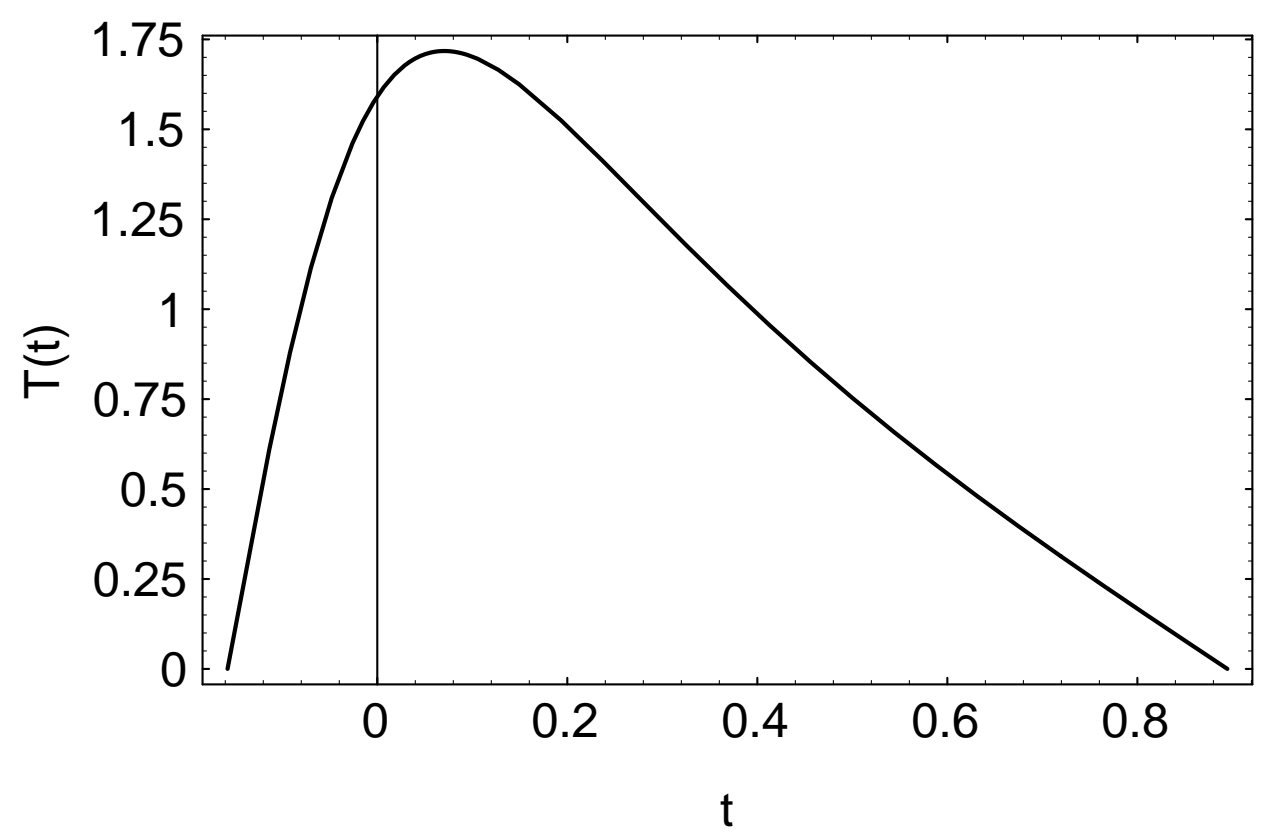

Figure 3: Temperature vs. $t$ for $v \gg 1$. 


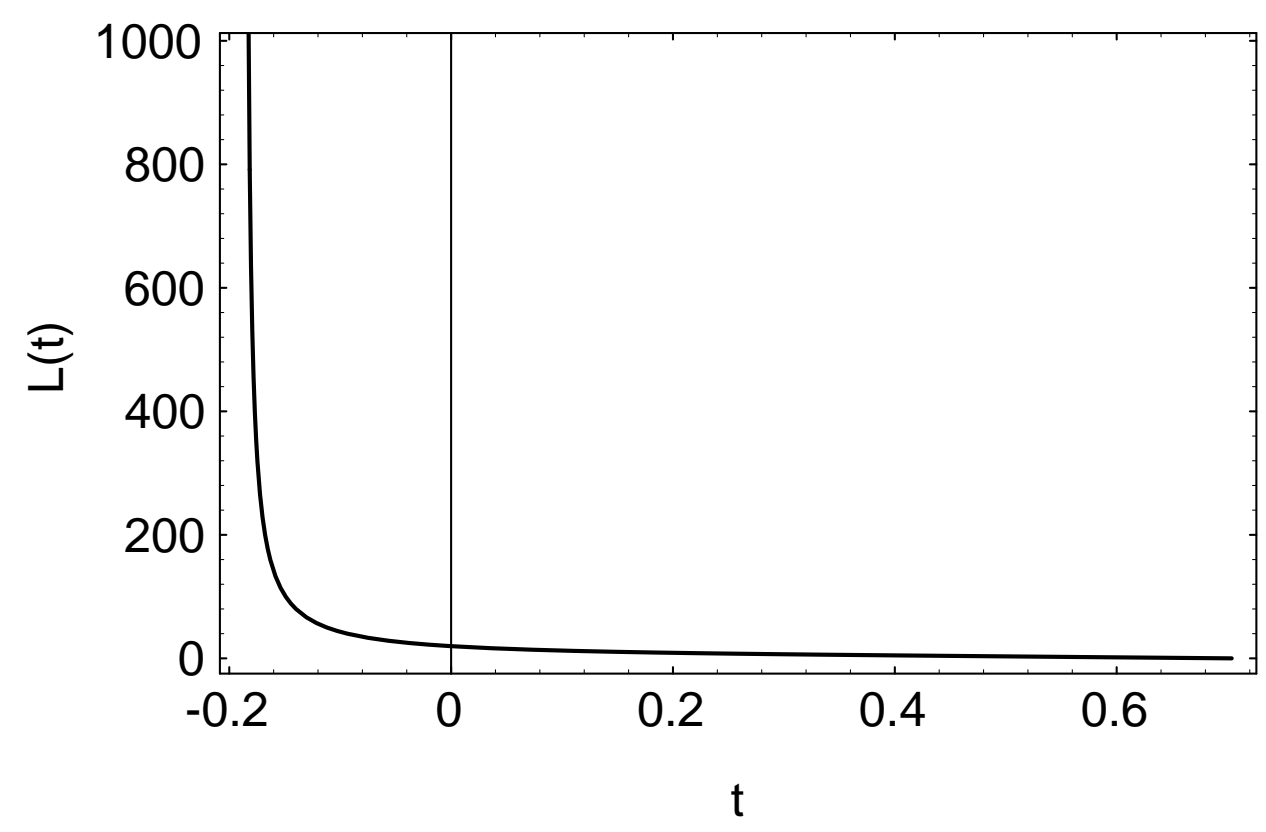

Figure 4: Scale factor vs. $t$.

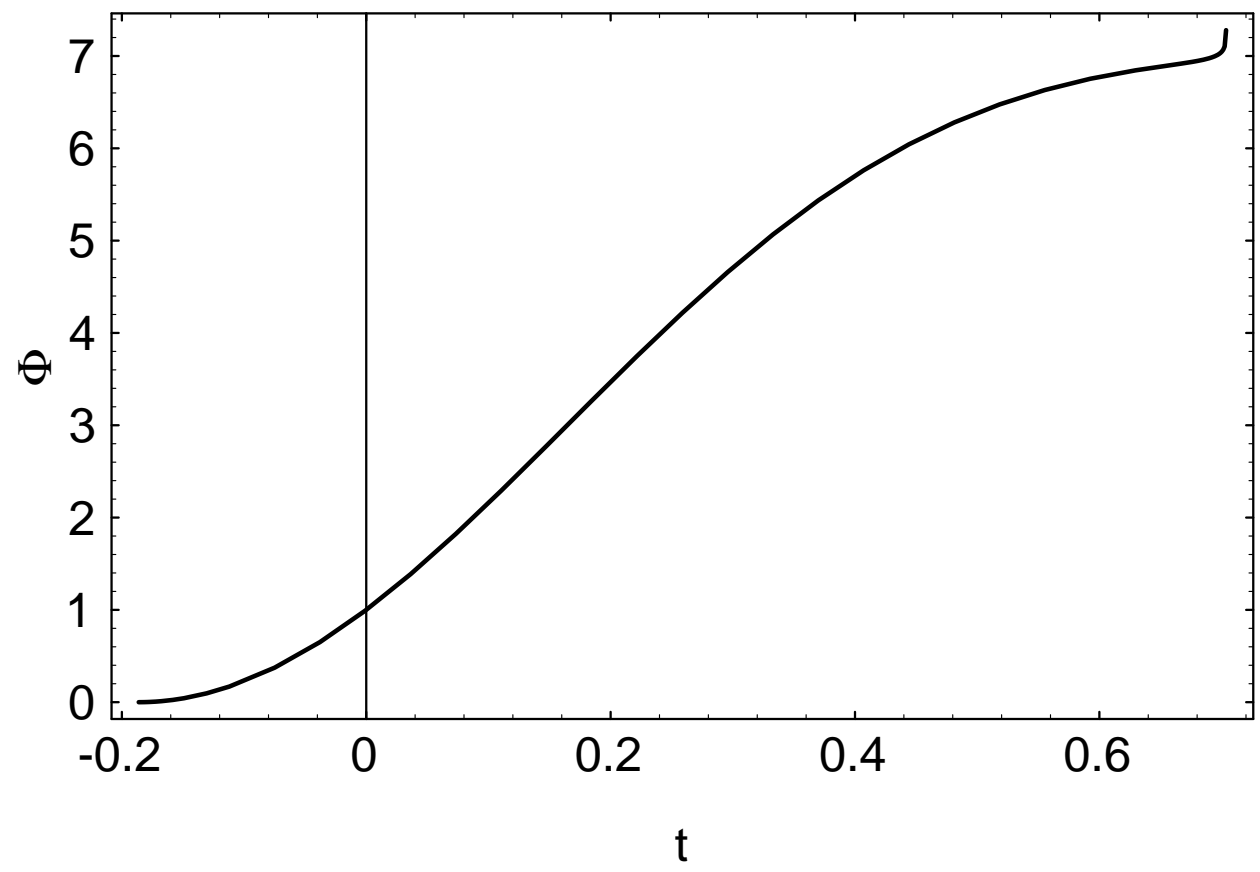

Figure 5: Dilaton field vs. $t$. 


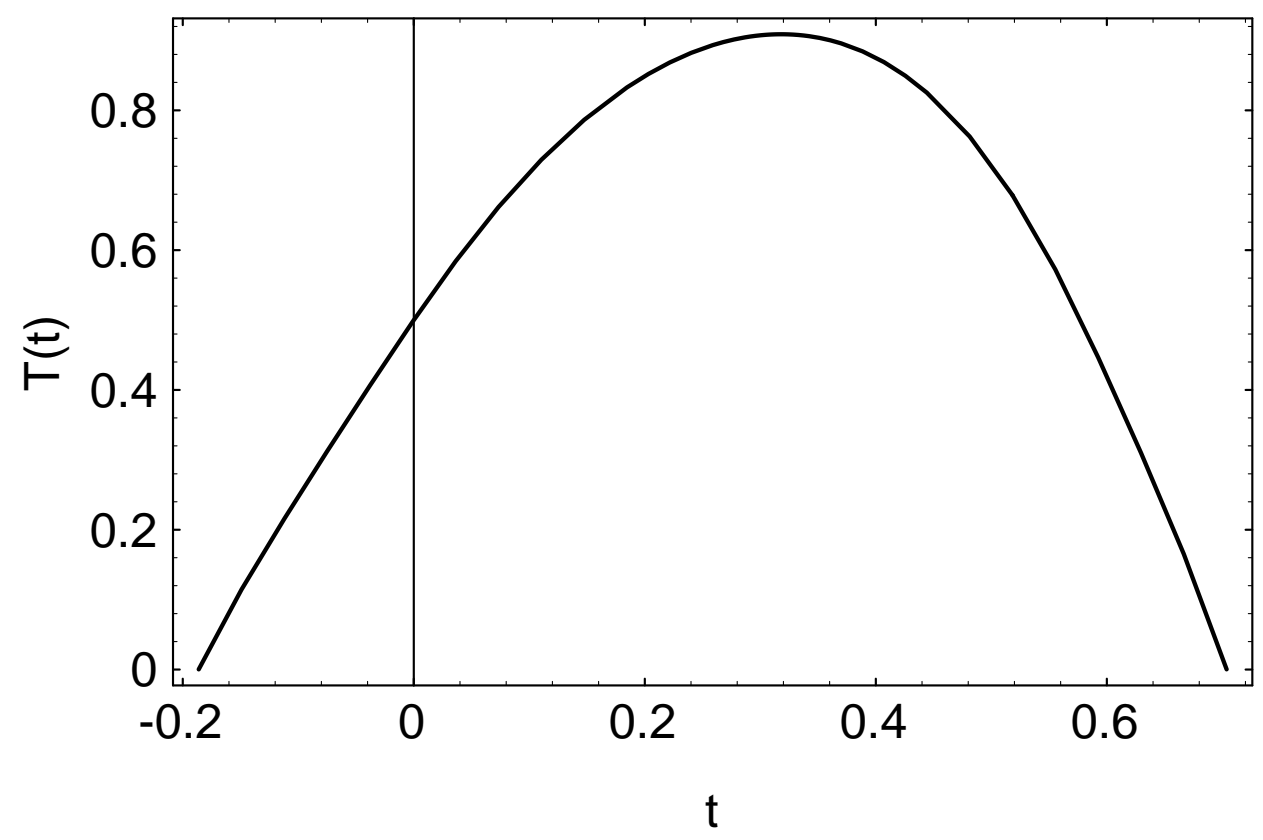

Figure 6: Temperature vs. $t$.

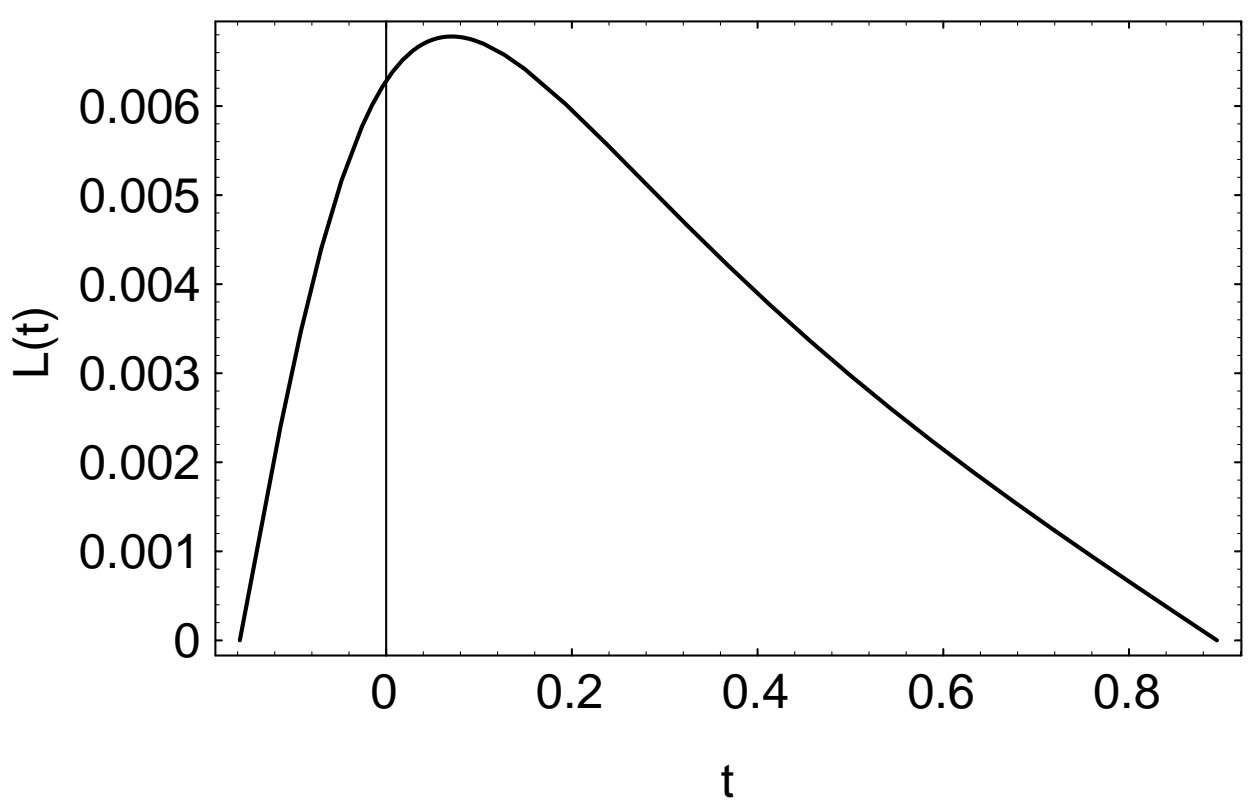

Figure 7: Scale factor vs. $t$ for the ultrastringy kind of solutions. 


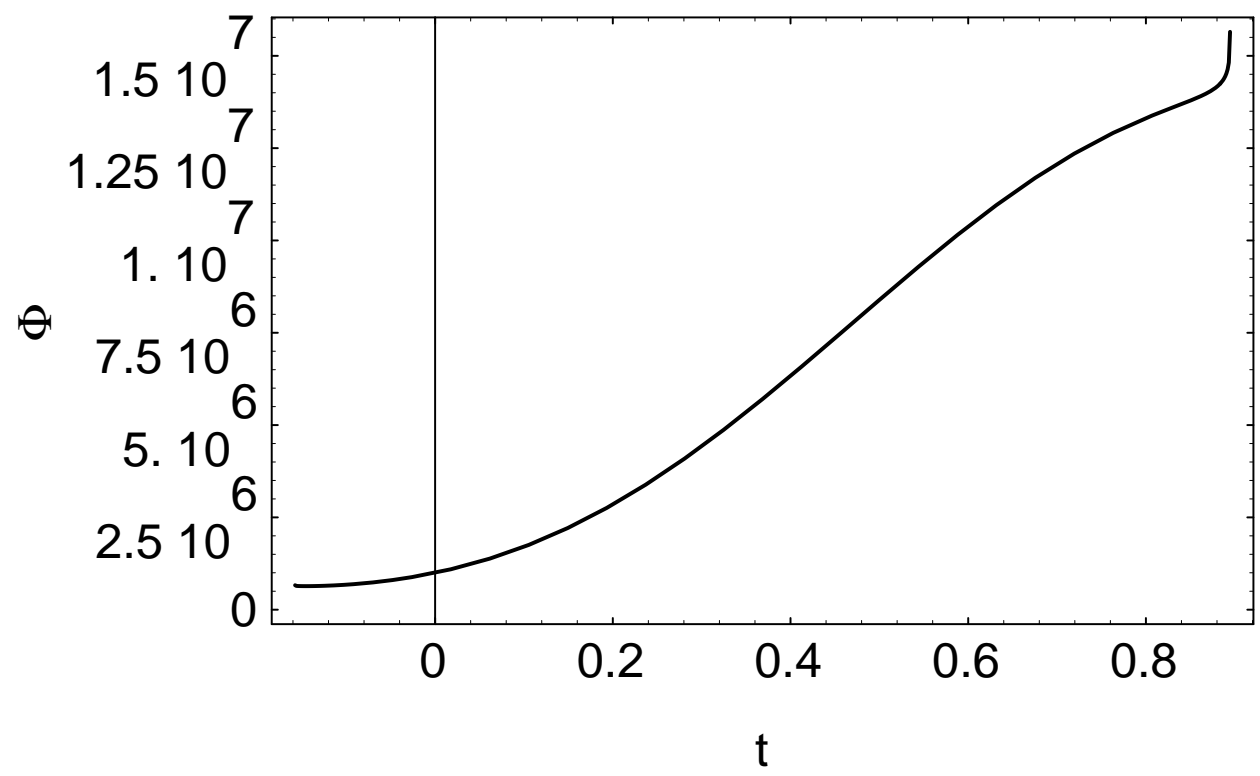

Figure 8: Dilaton field vs. $t$ for the ultrastringy kind of solutions.

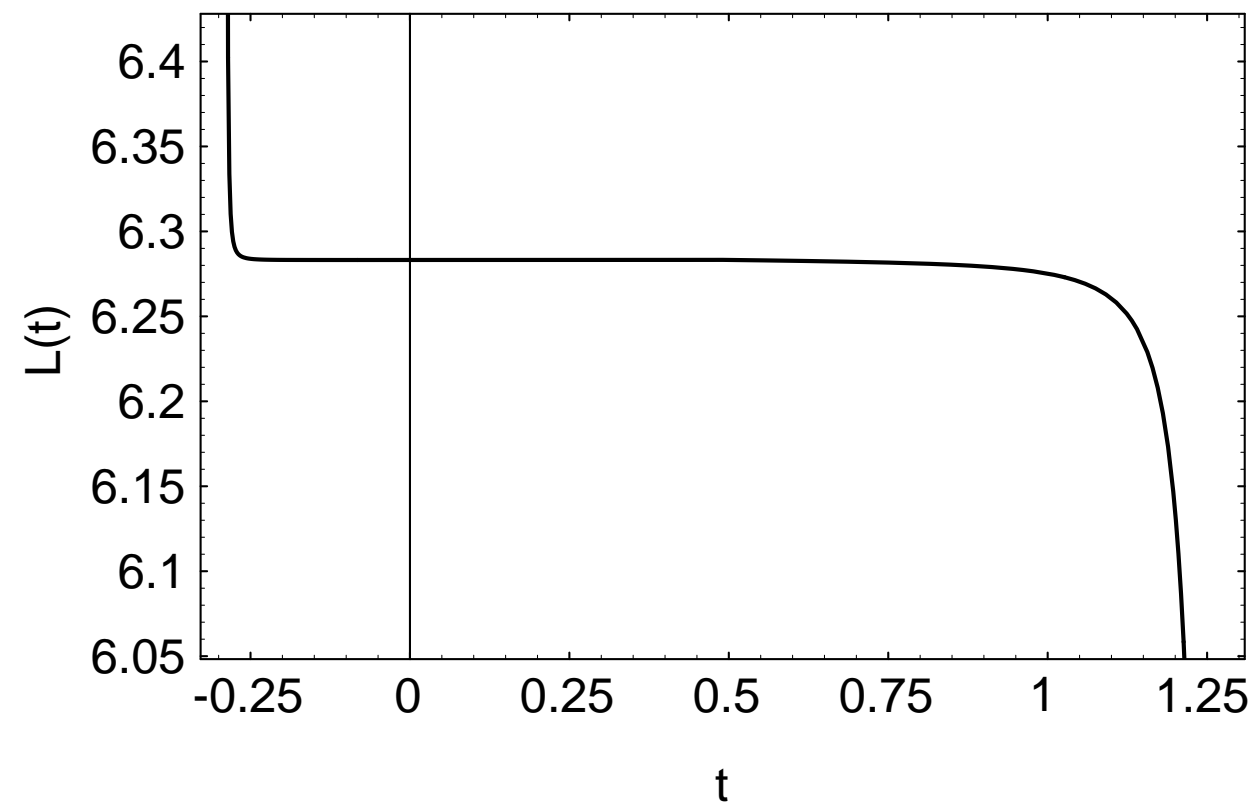

Figure 9: Scale factor vs. $t$ for the intermediate case. 


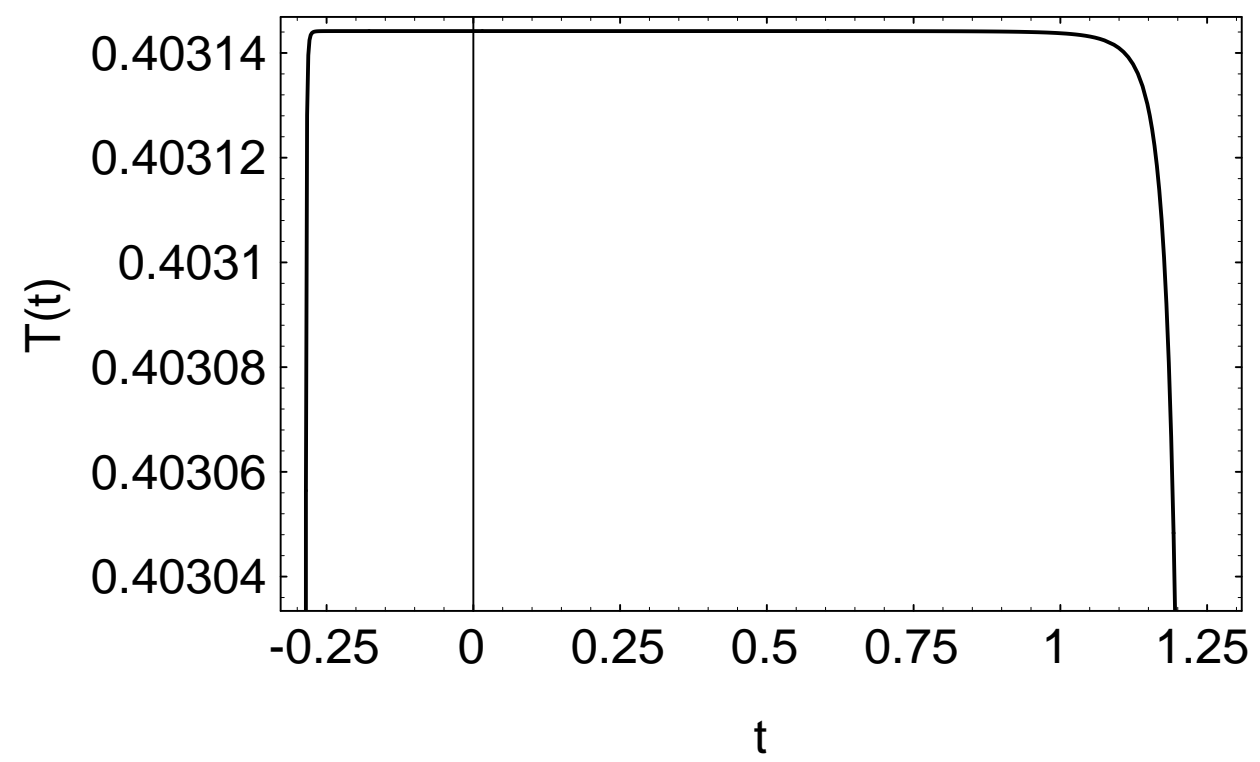

Figure 10: Temperature vs. $t$ for the intermediate case. 